\title{
Dental electives in a global pandemic - this is Newcastle calling!
}

\author{
Emma Robinson, Clinical Fellow in Oral Surgery, School of Dental Sciences, \\ Newcastle University, explains how a programme of virtual elective studies \\ were developed to counter the severe travel restrictions imposed during the \\ COVID-19 pandemic.
}

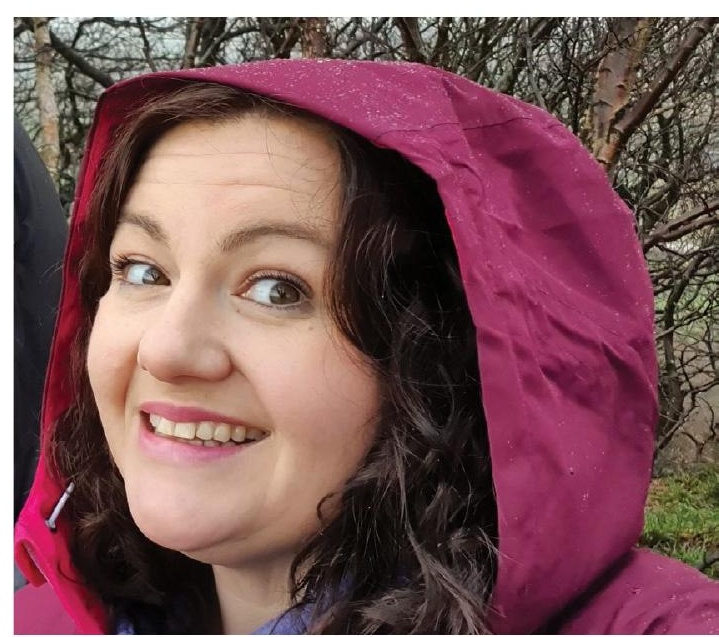

$\mathrm{I}$

loved my elective: I travelled to India, met amazing people, learnt about a different culture, and witnessed first-hand how healthcare is delivered in a country different from my own. It was a long time ago but I gained so much from the experience, and I believe it has shaped both the clinician and person I am today. So, in those dark days of 2020 when the pandemic started in the UK, whilst it was completely correct to cancel elective studies, it was also enormously sad. This sadness was echoed by the students, missing this opportunity, one which they had looked forward to for years. After the initial dust of the pandemic settled, I started to think about how some of the elements of the elective could be recreated in line with restrictions. What is the real 'essence' of an elective study? What are the intended learning outcomes from an elective period of study?

I spoke with colleagues at Newcastle and internationally and reflected upon experience and skills students receive. An elective period of study gives students autonomy over their studies, encouraging them to take responsibility for organising all aspects

\section{'An elective gives students autonomy over their studies'}

of the elective experience - from budgeting accommodation and travel to engaging with those they will work with. Students benefit from networks and friendships and broaden their outlook culturally, professionally and personally. These all will, in turn, shape the clinicians they become. I wanted this new elective to be student-led with opportunity for cultural exchange and personal growth.

With mentorship from colleagues, this idea evolved into a four-week online virtual exchange between Newcastle University, Universitas Indonesia and Fiji National University. The exchange was open to both BDS and ODHS (Oral and Dental Health Sciences) students. We split the students up into small groups of three/four students, ensuring there was a representative from each country within each group. The elective itself was split into two main parts. The first, about getting to know one another and learning about life and dentistry in different areas of the world. We wanted to encourage cultural exchange and time to get to know one another. The second part entailed working together on a joint project of their choice presenting this work in the form of a pre-recorded video. We asked the students to complete a reflective log that enabled us to get a sense of what our students enjoyed or found challenging and, from a process perspective, what had worked.

After the initial opening meeting held via Microsoft Teams, there was a deliberate 'hands off' approach, as it was more in the spirit of the 'Newcastle Elective' to allow students autonomy of decision and direction which we hoped would result in more meaningful and enriching endeavours. Further guidance and details of how the presentations would be marked was distributed. Students were asked to pre-record their ten-minute presentations and submit them before the final presentation meeting.

The presentations were shown at a mass meeting on a Saturday morning to work around three time zones. There were 11 amazing and varied presentations. Our students did not let us down; it was clear they had invested much time and enjoyed working with their equally inspirational international colleagues. The students chose an impressive range of subjects to focus on, from the impact of COVID19 on dentistry to comparative course structures, sustainability in dentistry and oral health initiatives. Many of them had been proactive, going out and speaking to experts in their chosen study areas to gain further insights.

All of the presentations were marked by staff and peers. A cash prize was available for the winning presentation from the North of England Odontological Society. There was originally only going to be a single winning group project but the standard was so high there was a need for a first runner up and a joint second runner up prize with no cash attached but plenty of kudos.

Feedback from reflective logs has been universally positive, with students highlighting their shared experiences and interests with students from other countries. They reflected upon learning about different cultures. It was hugely positive that students from all 

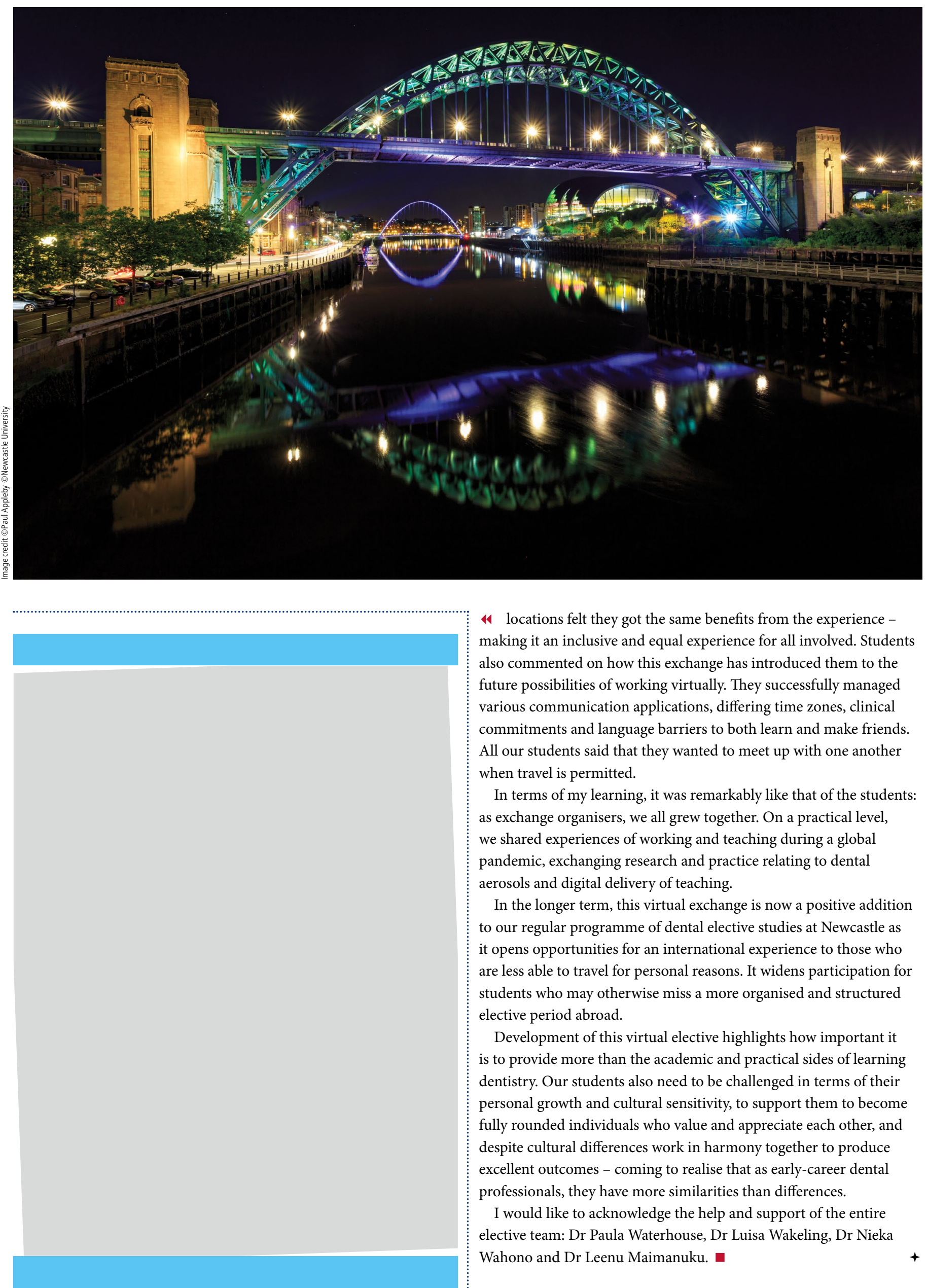

1 locations felt they got the same benefits from the experience making it an inclusive and equal experience for all involved. Students also commented on how this exchange has introduced them to the future possibilities of working virtually. They successfully managed various communication applications, differing time zones, clinical commitments and language barriers to both learn and make friends. All our students said that they wanted to meet up with one another when travel is permitted.

In terms of my learning, it was remarkably like that of the students: as exchange organisers, we all grew together. On a practical level, we shared experiences of working and teaching during a global pandemic, exchanging research and practice relating to dental aerosols and digital delivery of teaching.

In the longer term, this virtual exchange is now a positive addition to our regular programme of dental elective studies at Newcastle as it opens opportunities for an international experience to those who are less able to travel for personal reasons. It widens participation for students who may otherwise miss a more organised and structured elective period abroad.

Development of this virtual elective highlights how important it is to provide more than the academic and practical sides of learning dentistry. Our students also need to be challenged in terms of their personal growth and cultural sensitivity, to support them to become fully rounded individuals who value and appreciate each other, and despite cultural differences work in harmony together to produce excellent outcomes - coming to realise that as early-career dental professionals, they have more similarities than differences.

I would like to acknowledge the help and support of the entire elective team: Dr Paula Waterhouse, Dr Luisa Wakeling, Dr Nieka Wahono and Dr Leenu Maimanuku. 